Case Report

\title{
AGGRESSIVE MESENTRIC FIBROMATOSIS: A RARE CASE REPORT AND REVIEW OF LITERATURE.
}

\author{
Rohan Shetty ${ }^{1}$, Shubha Bhat ${ }^{2}$, Rajesh Ballal ${ }^{3}$, Pramod Makannavar ${ }^{4}$ \& Anil Kumar K.N. ${ }^{5}$, \\ ${ }^{1}$ Asst. Prof, Dept. of Surgery, ${ }^{2}$ Asst. Prof., Dept. of Pathology, ${ }^{3}$ Professor, Dept of Surgery, \\ ${ }^{4,5}$ Post Graduate, Dept. of Surgery, K.S. Hegde Medical Academy, Nitte University, Deralakatte, Mangalore - 575018 \\ Correspondence \\ Rohan Shetty \\ Asst. Professor, Dept. of Surgery, K.S. Hegde medical academy \\ NITTE University, Deralakatte, Mangalore - 575 018, Karnataka, India. \\ E-mail : shettyrohan@rediffmail.com
}

\begin{abstract}
:
Mesentric fibromatosis is a proliferative fibroblastic neoplasm of the small intestinal mesentery with varied clinical presentation. Giant mesentric fibromatosis is uncommon and its rarity poses a diagnostic and therapeutic challenge. This paper presents a recurrent aggressive fibromatosis in a 38 year old male patient, who had initially undergone a laparotomy outside for mass abdomen but only pus was evacuated and definitive diagnosis was not made.
\end{abstract}

Keywords : Mesenteric fibromatosis, mass abdomen, abdominal abscess, benign neoplasms.

Introduction:

Fibromatosis are heterogenous group of condition which includes mesentric fibromatosis. Mesentric fibromatosis are benign proliferation of mesentric tissue that tends to recur without distant metastasis ${ }^{1}$. These tumors are histologically benign but they behave like malignant tumors. They might invade locally and recur after excision ${ }^{2}$. Giant mesentric fibromatosis are uncommon by itself ( 2-4 cases/million/year ). Some cases may be associated with other pathologies like, association with Gardener's syndrome (3-45\%), and Familial colonic polyposis ( $10 \%)^{3}$. Most reported cases have been associated with Gardener's syndrome, previous trauma and prolonged estrogen intake ${ }^{4}$. However primary mesentric fibromatosis, without any predisposing factors or association is also known. Patients presents to hospital with mass effects or obstruction of surrounding structures like small intestine and ureters ${ }^{5}$.

\begin{tabular}{|c|}
\hline Access this article online \\
\hline Quick Response Code \\
\hline
\end{tabular}

This report presents a case of aggressive mesentric fibromatosis in a young male who presented with history of pain abdomen, abdominal distension and previous history of laparotomy, which was managed in Department of surgery in K.S.Hegde hospital, Mangalore, India.

\section{Case Report :}

A 38 year old male presented with history of abdominal pain and distension of abdomen since 2 months. Patient also had history of previous laparotomy 1 year back for mass abdomen. Operative notes showed, intra abdominal abscess adjacent to the splenic flexure of colon and root of the mesentery without any other abnormality. Pus was evacuated and definitive histopathology was not possible then. Now, on examination patient had large mobile mass per abdomen, occupying most of the abdomen and a large laparotomy scar was also seen. Laboratory investigations were within normal limits. CT scan showed large mass lesion between anterior abdominal wall and mesentery measuring $11.8 \times 11.3 \times 12.9 \mathrm{~cm}$ which was surrounding by bowel loops (Fig 1,2). USG guided FNAC was inconclusive. With clinical diagnosis of GIST, patient underwent laparotomy. Intra operatively, large mass at the root of jejunal mesentery with loop of jejunum adherent to it was seen, along with small bowel adhesion due to previous surgery. Patient underwent resection of the tumor along with resection of $90 \mathrm{~cm}$ of jejunum and proximal ileum with end to end anastamosis (Fig 3). Patient recovered well without any post operative complications. Histopathology 


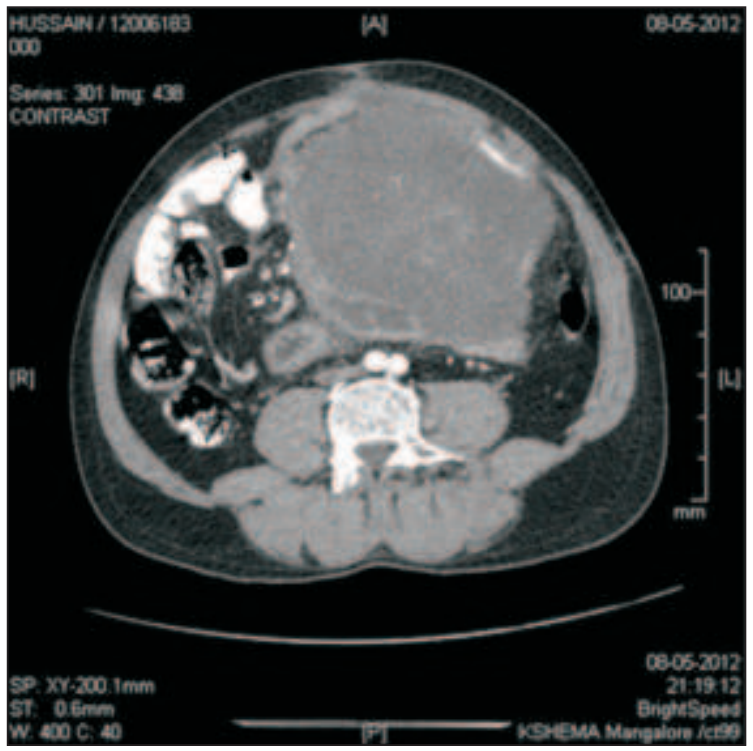

Fig 1 : CT scan picture showing large mass lesion in the mesentery surrounded by small bowel loops.

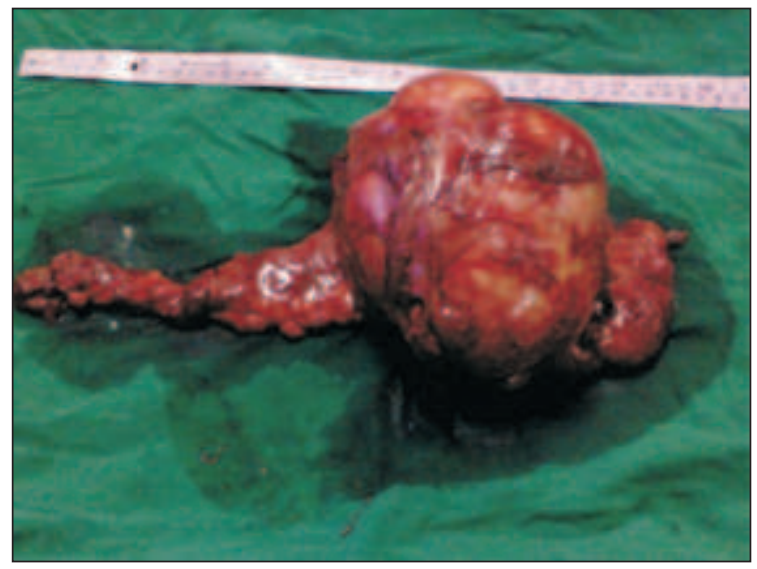

Fig 3: Large mesentric mass measuring about $15 \times 12 \mathrm{~cm}$ with approximately $90 \mathrm{~cm}$ of jejunum adherent to it.

showed moderate cellularity with elongated slender spindle shaped cells. Stroma showed few eosinophilic collagen fibers. Thin walled vessels were seen with perivascular hyalinisation. Masson trichrome staining was negative for smooth muscle. Sections studied from jejunum overlying tumor showed mildly flattened villi, lamina propria showed lymphoplasmacytic infiltrate. Features were suggestive of mesentric fibromatosis (Fig 4). Immunohistochemistry was done, which stained negative for CD 117 and CD34. Post operatively colonoscopy was

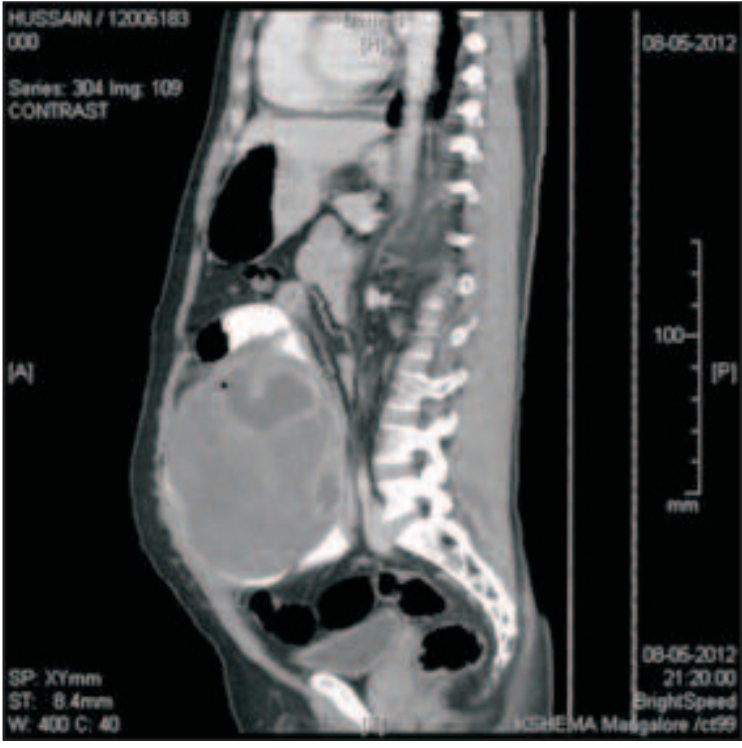

Fig 2 : CT scan sagital view.

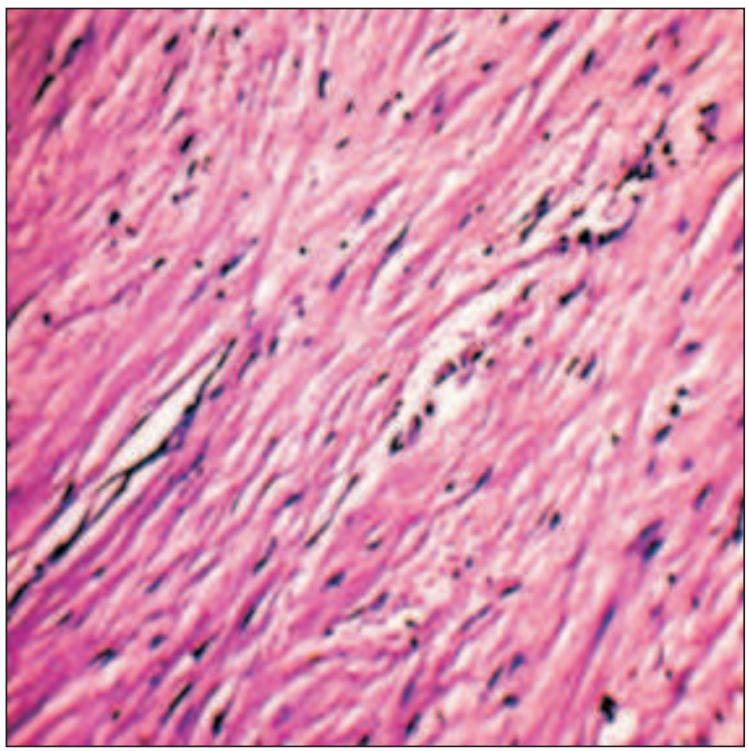

Fig4 : Microscopic picture showing spindle shaped cells with stroma containing eosinophilic collagen.

done which was normal and ruled out association with FAP syndrome.

\section{Discussion :}

Mesenteric fibromatosis are uncommon and often presents a diagnostic challenge. Its biological behavior is intermediate between benign fibrous tissue proliferation and Fibrosarcoma. The differential diagnosis are, a bland spindle cell tumor involving the GIT and from the mesentery includes GIST, fibromatosis and inflammatory myofibroblastic tumor ${ }^{6}$. Even though mesentric 
fibromatosis tends to be aggressive, there is considerable variability in their growth rate during the course of the disease. Usually they are characterized by initial rapid growth followed by stability or even regression. Commonly, patient presents with vague pain abdomen and distension. However, because of their ability to infiltrate they can present with intestinal obstruction, ischemia, perforation, hydronephrosis ${ }^{7,8}$. In spite of this overall 10 year survival rate for mesenteric fibromatosis can be as high as $60-70 \%{ }^{9}$.

The radiological features of these tumors are variable, there are no specific imaging features to distinguish between mesenteric fibromatosis and other mass lesions in the abdomen. CT scan and MRI scan are useful for evaluating the size of the tumor and involvement of adjacent structures ${ }^{10}$.

Histopathological examination is essential for diagnosis. On gross examination tumor is well circumscribed and white and coarsely trabeculated on cross section. Microscopically mesenteric fibromatosis is characterized by a spatially homogenous proliferation of wavy spindle cells without atypia, associated with collagen among dilated vessels. The mitotic count is relatively low with no evidence of necrosis and nuclear differentiation ${ }^{11}$.

Immunohistochemistry can differentiate mesenteric fibromatosis from GIST and inflammatory myofibroblastic tumors. Mesenteric fibromatosis positively stain for nuclear beta catenin and negative for CD 117 and CD34.Sometimes weak positivity or focal positivity for CD117 may be seen. GIST will stain positive for CD117 and CD34 but negative for beta catenin. Inflammatory myofibroblastic tumor stain negative for all the above three $^{12}$

\section{References}

1. Mohammed Khalid Mirza Gari, Salman Yousuf Guraya, Amir Mounir Hussein, Moustafa Mahmoud Nafady Hego. Giant mesenteric fibromatosis: Report of a case and review of the literature. World J Gastrointest Surg 2012 March 27; 4(3): 79-82

2. Hung TS, Yu JC, Gao HW, Liu Yc, Hseih CB. Spontaneous isolated pedunculated mesenteric fibromatosis in a patient with acute abdominal pain and persistent fever. J Med Sci 2004:24:227-30.

3. Marone U, Amore A, Pezzullo F, Mozzillo N. Giant desmoid tumour of the abdominal wall associated with familial adenomatous polyposis.
Many authors believe, complete surgical excision with a margin of uninvolved tissue is the treatment of choice for mesenteric fibromatosis. Unfortunately, radical surgery is not always a straight forward procedure because of the extent and invasiveness of the tumor. Involvement of base of mesentery and major portion of mesenteric blood supply makes surgery difficult and increases morbidity and mortality ${ }^{13}$. Debulking has no role, as it leads to more aggressive and infiltrative growth ${ }^{14}$. Inoperable growth should only be biopsied and other options of treatment need to be planned.

NSAIDs like Sulindac used post operatively and antiestrogen therapy with tamoxifen and testolactone can be used for many years. For clearly inoperable cases, cytotoxic chemotherapy, especially doxorubicin based or low dose vinblastin and methotrexate has been proposed. The evidence in the literature supports the opinion that both cytotoxic and non cytotoxic chemotherapy are effective against fibromatosis. However, lack of sufficient patients and randomized trials compromises the validity of the reported results ${ }^{15}$.

In our patient, he was not put on any medications after surgery and was asked to be on regular follow up.

\section{Conclusion :}

There are no designed treatment protocols for mesentric fibromatosis. All modalities including surgery, chemotherapy radiation therapy are required in some cases. The rarity of these cases in high volume oncological centres has limited the ability to study the disease. Probably it is worthwhile to follow up all reported cases prospectively and draw some conclusion.

Tumori 2003; 89: 331-332.

4. Karakousis CP, Berjian RA, Lopez R, Rao U. Mesenteric fibromatosis in Gardner's syndrome. Arch surg 1978; 113: 998-1000

5. SY Luk, KH Fung, SH Fung. Mesenteric Fibromatosis: An Uncommon Cause of Abdominal Pain. J Hong Kong Col Radiol 2010; 13: 218-21

6. Colombo P, Rahal D, Grizzi F, Quagliuolo V. Roncalli M : Localized intraabdominal fibromatosis of the small bowel mimicking a gastrointestinal stromal tumor: a case report. World J Gastroenterol 2005, 11: 5226-5228. 
7. Holubar S, Dwivedi AJ, O'Connor J: Giant mesenteric fibromatosis presenting as small bowel obstruction. Am Surg 2006, 72: 427-9.

8. Collins D, Myers E, Kavanagh D, Lennon G, McDermott E: Mesenteric desmiod tumor causing ureteric obstruction. International J Uro 2008, 15: 261-262.

9. Stoidis et al.: Surgical treatment of giant mesenteric fibromatosis presenting as a gastrointestinal stromal tumour: a case report. Journal of medical case reports 2010: 4:314.

10. Casillas J,Sais GJ, Greve JI, Iparraguirre MC, Morillo G. Imaging of intra\& extraabdominal desmiod tumours. Radiographics 1991; 11:959-68.

11. Rodriguez JA, Guarda LA, Rosai J. Mesenteric fibromatosis with involvement of gastrointestinal tract. A GIST simulator: a study of 25 cases. Am J Clin Pathol. 2004;121:93-8.
12. Yantiss RK, Spiro IJ, Compton CC, Rosemberg AE: Gastrointestinal Stromal Tumor versus intra-abdominal fibromatosis of the bowel wall. Am J Surg Pathol 2000, 24:947-957.

13. Melis M, Zager JS, Sondak Vk: Multimodality management of desmoid tumors: how important is a negative surgical margin? J Surg Oncol 2008, 98: 594-602.

14. Latchford AR, Sturt NJH, Neale K, Rogers PA, Philips Rk: A ten year review of surgery for desmoid disease associated with familial adenomatous polyposis. Br J Surg 2006, 93: 1258-64.

15.J Janinis, M Pattriki, L Vini, G Aravantinos, J S Whelan. The pharmacological treatment of aggressive fibromatosis: a systemic review. Annals of Oncology 2003; 14: 181-190. 